\title{
A NOTE ON DARK SOLITONS IN NONLINEAR COMPLEX GINZBURG-LANDAU EQUATIONS
}

\author{
AGUSTIN TOMAS BESTEIRO
}

\begin{abstract}
We analyze the existence of dark solitons in nonlinear complex Ginzburg-Landau equations. We prove existence results concerned with the initial value problem for these equations in Zhidkov spaces using a new approach with splitting methods.
\end{abstract}

MSC 2010. 47J35.

Key words. Ginzburg-Landau equation, splitting methods, well posedness.

\section{REFERENCES}

[1] I.S. Aranson and L. Kramer, The world of the complex Ginzburg-Landau equation, Rev. Modern Phys., 74 (2002), 99-143.

[2] A.T. Besteiro and D.F. Rial, Global existence for vector valued fractional reactiondiffusion equations, preprint (2018), arXiv:1805.09985.

[3] J.P. Borgna, M. De Leo, D. Rial and C. Sanchez de la Vega, General splitting methods for abstract semilinear evolution equations, Commun. Math. Sci., 13 (2015), 83-101.

[4] T. Cazenave and A. Haraux, An introduction to semilinear evolution equations, in Oxford Lecture Series Mathematics and Applications, Vol. 13, Clarendon Press, 1999.

[5] M. De Leo, D. Rial and C. Sanchez de la Vega, High-order time-splitting methods for irreversible equations, IMA J. Numer. Anal., 36 (2015), 1842-1866.

[6] N. Efremidis, K. Hizanidis, H.E. Nistazakis, D.J. Frantzeskakis and B.A. Malomed, Stabilization of dark solitons in the cubic Ginzburg-Landau equation, Phys. Rev. E, 63 (2000), 7410-7414.

[7] K.J. Engel and R. Nagel, One-parameter semigroups for linear evolution equations, Graduate Texts in Mathematics, Vol. 194, Springer-Verlag, New York, 1999.

[8] C. Gallo, Schrödinger group on Zhidkov spaces, Adv. Differential Equations, 9 (2004), 509-538.

[9] J. Ginibre and G. Velo, The Cauchy problem in local spaces for the complex GinzburgLandau equation. I. Compactness methods, Phys. D, 95 (1996), 191-228.

[10] J. Ginibre and G. Velo, The Cauchy problem in local spaces for the complex GinzburgLandau equation. II. Contraction methods, Comm. Math. Phys., 187 (1997), 45-79.

[11] Y.S. Kivshar and B. Luther-Davies, Dark optical solitons: physics and applications, Phys. Rep., 298 (1998), 81-197.

The authors would like to thank CONICET for supporting this research and the referee for his helpful comments and suggestions.

DOI: $10.24193 /$ mathcluj.2020.1.02 
[12] P. Zhidkov, The Cauchy problem for a nonlinear Schrödinger equation (in Russian), Joint Inst. for Nuclear Research, Dubna (USSR), Lab. of Computing Techniques and Automation, P5-87-373 (1987), 1-18.

Received February 22, 2019

Accepted April 14, 2019
Instituto de Matemática Luis Santaló CONICET-UBA

Ciudad Universitaria, Pabellón I (1428)

Buenos Aires, Argentina

E-mail: abesteiro@dm.uba.ar 